\title{
Valores hematológicos em eqüinos naturalmente infectados por estrongilídeos
}

\author{
Hematologic parameters in horses naturally infected with strongyles
}

\author{
Peter Reichmann; Júlio Augusto Naylor Lisboa'; Mara Regina Stipp Balarin²; \\ Ademir Benedito da Luz Pereira²
}

\begin{abstract}
Resumo: Com objetivo de verificar as possíveis alterações hematológicas relacionadas ao parasitismo intestinal em eqüinos selecionaram-se 84 animais (42 machos, 42 fêmeas), mestiços, com idade entre 2 e 15 anos, utilizados em trabalho diário de tração urbana, com histórico de emagrecimento e menor resistência ao esforço físico, diagnosticando-se apenas elevada infecção natural por estrongilídeos. A contagem de ovos por gramas de fezes (OPG) mostrou uma variação de 350 a 6.500 OPG (Md = 1.425 OPG) caracterizando um grau considerável de infecção por formas adultas de estrongilídeos, sendo o quadro hematológico representado pelos valores médios a seguir; Eritrócitos: 6,43

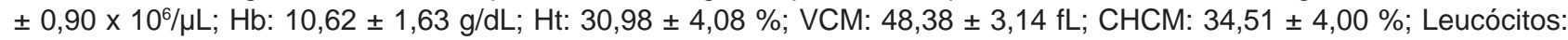

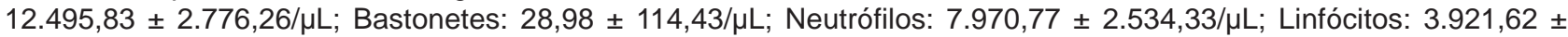

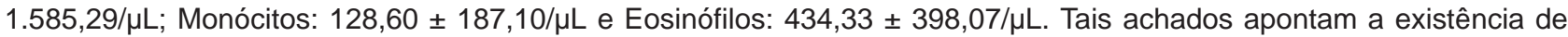
leve leucocitose por neutrofilia, assim como uma tendência do quadro eritrocitário em se manter próximo aos limites inferiores de normalidade considerados para a espécie. Não se observou, por fim, qualquer alteração quanto aos valores de eosinófilos, contradizendo em parte o conceito de que a eosinofilia deva acompanhar os quadros de parasitismo intestinal na espécie eqüina.
\end{abstract}

Palavras-chave: Eqüinos, valores hematológicos, infecção por estrongilídeos.

\begin{abstract}
In order to verify the possible hematologic alterations related to intestinal parasitism in horses, 84 mixed breed animals (42 males, 42 females) were selected, with age varying from 2 to 15 years, used in the daily routine work of urban traction, with history of weight loss and less resistance to physical effort, and with a diagnoses of intense natural infection with strongyles. The fecal egg count per grams of feces (EPG) showed a variation from 350 to 6.500 EPG (Md 1.425 EPG) characterizing a considerable degree of infection with adult strongyles. The hematologic parameters were erithrocytes

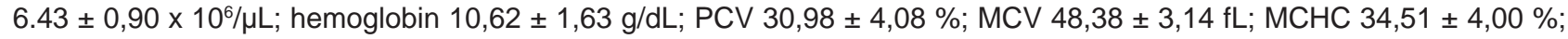
leucocytes $12.495 \pm 2.776,26 / \mu \mathrm{L}$; band neutrophils $28,98 \pm 114,43 / \mu \mathrm{L}$; mature neutrophils $7.970,77 \pm 2.534,33 / \mu \mathrm{L}$; lymphocytes $3.921 \pm 1.585,29 / \mu \mathrm{L}$; monocytes $128,60 \pm 187,10 / \mu \mathrm{L}$ and eosinophils $434,33 \pm 398,07 / \mu \mathrm{L}$. These values showed a leucocytosis with neutrophilia, as well as a tendency of erythrocytic values in staying close to the normal inferior limits of the reference range for the specie. No alterations regarding eosinophylic values were observed, thus partly contradicting the concept that eosinophilia should occur with intestinal parasitism in horses.
\end{abstract}

Key words: Horse, hematologic parameters, infection with strongyles.

\section{Introdução}

As infecções por estrongilídeos em eqüinos podem estar associadas a várias alterações no hospedeiro, onde importância maior no caso dos grandes estrôngilos é dada para a arterite tromboembólica da artéria mesentérica cranial e o comprometimento da circulação intestinal local causadas pela migração durante a fase larvar. Nos pequenos estrôngilos é dada importância semelhante à ação sobre a parede e mucosa intestinal tanto da forma larvar, quando encistada ou deixando os cistos (ciatostomíase larvar), como da forma adulta que se alimenta desta mucosa, deixando pequenas feridas ou ulcerações, resultando em hemorragias e extravazamento de proteinas plasmáticas para a luz intestinal. Apesar disto, as infecções naturais por estrongilídeos em eqüinos são freqüentemente subclínicas, ou podem resultar em pêlo opaco, emagrecimento e menor tole- rância ao esforço físico. Paralelamente, alterações hematológicas como anemia e leucocitose associadas ou não à eosinofilia têm sido relatadas como conseqüência do parasitismo intestinal por estrongilídeos induzido experimentalmente em eqüinos (AMBORSKI et al., 1974; PATTON; DRUDGE, 1977; KLEl et al., 1982; DENNIS et al., 1992).

O presente trabalho teve por objetivo verificar as alterações hematológicas relacionadas ao parasitismo intestinal em eqüinos naturalmente infectados por estrongilídeos.

\section{Materiais e Métodos 2.1 Animais}

Foram utilizados 84 eqüinos, dos quais 42 machos e 42 fêmeas, mestiços, com idade oscilando entre $2 \mathrm{e}$

\footnotetext{
${ }^{1}$ Departamento de Clínicas Veterinárias, Universidade Estadual de Londrina, Cx.P. 6001, 86051-970 Londrina - PR

2 Departamento de Medicina Veterinária Preventiva, Universidade Estadual de Londrina
} 
15 anos (a maioria entre 3 e 9 anos; $n=76$ ), utilizados em trabalho diário de tração urbana e encaminhados ao serviço ambulatorial de Clínica Médica de Grandes Animais do Hospital Veterinário da Universidade Estadual de Londrina - PR, com histórico de emagrecimento, menor resistência ao esforço físico e manejo nutricional e sanitário inadequados, diagnosticando-se apenas elevada infecção natural por estrongilídeos.

\subsection{Colheita de material e processamento 2.2.1. Fezes}

A partir de fezes colhidas diretamente da ampola retal realizou-se a contagem de ovos por grama de fezes (OPG) através da técnica de Gordon e Whitlock modificada, com sensibilidade para um mínimo de 50 OPG.

\subsubsection{Sangue}

O sangue foi colhido por venopunção da jugular cervical em frascos contendo EDTA.

As variáveis hematológicas foram obtidas conforme os métodos clássicos preconizados (JAIN, 1986). A contagem de eritrócitos e de leucócitos totais foi realizada com o uso de câmara de Newbauer e a contagem diferencial dos leucócitos estabelecida a partir de esfregaços sangüíneos corados pelo método do corante hematológico rápido (Panótico).

A hemoglobina foi dosada por meio do método da cianometahemoglobina com leitura espectrofotométrica (Espectrofotômetro - CELM E-225D).

A determinação do volume globular foi realizada por meio da centrifugação do sangue com o auxílio de tubos capilares (Centrífuga Microhematócrito - FANEM Mod. 210 I.E.C.).

Os valores de volume corpuscular médio (VCM) e de concentração de hemoglobina corpuscular média (CHCM) foram calculados segundo as fórmulas clássicas recomendadas por Jain (1986).

\subsection{Análise estatística}

Realizou-se um estudo estatístico descritivo com determinação de medidas de tendência central e de dispersão para as variáveis quantitativas estudadas (BERQUÓ et al., 1980).

\section{Resultados e Discussão}

Os valores de OPG mínimos e máximos verificados foram 350 e 6.500 respectivamente, com média aritmética de $1.740,48 \pm 1.212,77$ e mediana 1.425 .

Os valores hematológicos estão relacionados na Tabela 1.

Apesar de valores de OPG não serem um retrato fiel do número de parasitas presentes no hospedeiro (UHLINGER, 1993), os valores obtidos neste trabalho podem ser considerados significativos de infecção elevada por formas adultas de estrongilídeos devido aos sintomas de baixo rendimento e emagrecimento apresentados pelos eqüinos.

Os achados do hemograma, quando comparados a valores de referência para a espécie eqüina (JAIN, 1986) e em especial a valores referentes a eqüinos sem raça definida usados em tração urbana no Brasil (FARIA et al., 1996), apontaram no eritrograma uma tendência em se manter próximos dos limites inferiores de normalidade. O leucograma apresentou leve leucocitose devido a uma neutrofilia e valores normais de linfócitos e eosinófilos (Tabela 1).

Tabela 1 - Valores médios (X), desvio padrão (s) e mediana (Md) obtidos para as variáveis hematológicas em eqüinos $(n=84)$ naturalmente infectados por estrongilídeos.

\begin{tabular}{ll}
\hline & \pm \\
& \pm \\
& \pm \\
& \pm \\
& \pm \\
& \pm \\
& \pm \\
& \pm \\
& \pm \\
& \pm \\
& \pm \\
\end{tabular}

Estes dados diferem dos observados por Sartori Filho et al. (1993), que não encontraram alterações hematológicas associadas à infecção natural por estrongilídeos, sendo porém os dados destes autores obtidos em eqüinos sem sinais clínicos de verminose.

Em poneis com sintomatologia decorrente de infecções experimentais por formas larvares $\left(L_{3}\right)$ de Strongylus vulgaris foram relatadas leucocitose por neutrofilia e eosinofilia, estas mais ou menos evidentes de acordo com a dose infectante e a intensidade da sintomatologia apresentada, associadas a uma leve anemia do tipo normocrômica e normocítica (AMBORSKI et al., 1974; PATTON; DRUDGE, 1977; KLEI et al., 1982; DENNIS et al., 1992). Já em casos de infecções naturais por pequenos estrôngilos, onde também foi relatada leucocitose, as presenças de anemia e eosinofilia dependem da intensidade e do tipo do quadro clínico apresentado (GILES et al., 1985; REINEMEYER, 1986). Estes dados podem ser em parte comparados aos obtidos neste trabalho, onde se verificou apenas uma leve leucocitose por neutrofilia e uma tendência à ocorrência de anemia, provavelmente devidos a uma ação não tão intensa dos parasitas sobre o hospedeiro, evidenciada por uma sintomatologia mais branda e pouco específica de menor resistência ao esforço físico e emagrecimento.

A eosinofilia não foi constatada nos animais estudados, contradizendo em parte o conceito de que a mesma 
deva acompanhar os quadros de endoparasitismo em eqüinos. Tal aparente contradição pode ser explicada pelo fato de a eosinofilia ocorrer particularmente como uma conseqüência da migração dos parasitas em fase larvar após infecções experimentais maciças puras, principalmente por Strongylus vulgaris (AMBORSKI et al., 1974; PATTON; DRUDGE, 1977; KLEI et al., 1982; DENNIS et al., 1992), e de que no casos das infecções naturais, mistas por excelência, os ciatostomíneos representam a grande maioria dos parasitas presentes no trato intestinal quando comparados aos grandes estrôngilos (SARTORI FILHO et al., 1993; LUZ PEREIRA et al., 1994).

\section{Referências}

AMBORSKI, G.F.; BELLO, T.R.; TORBERT, B.J. Host response to experimentally induced infections of Strongylus vulgaris in parasite-free and naturally infected ponies. Am. J. vet. Res., v.35 p.1181-1188, 1974.

BERQUÓ, E.S.; SOUZA, J.P.M.; GOTLIEB, L.B. Bioestatística. EPU, São Paulo, 1980. 325p.

DENNIS, V. et al. Immune response of pony foals during repeated infections of Strongylus vulgaris and regular ivermectin treatments. Vet. Parasitol., v.42 p.83-99, 1992.

FARIA, E.P. et al. Avaliação hematimétrica em eqüinos sem raça definida na região metropolitana de Belo Horizonte - MG. Resultados parciais. In: CONGRESSO BRASILEIRO DE MEDICINA VETERINÁRIA, 24, Goiânia. Anais ... Goiânia, p.11-12, 1996.
GILES, J.L.; URQUHART, K.A.; LONGSTAFFE, J.A. Larval cyathostomiasis (immature trichonema-induced enteropathy): A report of 15 clinical cases. Equine Vet. J., v.17 n.3 p.196201, 1985.

JAIN, N.C. Schalm's veterinary hematology. 4. ed. Philadelphia: Lea \& Febiger, 1986. 1221p.

$\mathrm{KLEI}, \mathrm{T}$.R. et al. Morphologic and clinicopathologic changes following Strongylus vulgaris infections of immune and nonimmune ponies. Am. J. Vet. Res., v.43, n.7, p.300-1307, 1982

LUZ PEREIRA, A.B. et al. Efcácia a campo do mebendazole, oxibendazole, pamoato de pirantel e doramectin contra pequenos estrongilídeos (Cyathostominae) de eqüinos. Rev. Bras. Parasitol. Vet., v.3 n.2 p.93-97, 1994.

PATTON, S.; DRUDGE, J.H. Clinical response of pony foals experimentally infected with Strongylus vulgaris. Am. J. Vet. Res., v.38, n.12 p.2059-2066, 1977.

REINEMEYER, C.R. Small strongyles - Recent advances. Vet. Clin. North Am. Equine Pract., v.2, n.2 , p.281-312, 1986.

SARTORI FILHO, R.; AMARANTE, A.F.T.; OLIVEIRA, M.R. Efeito de medicações anti-helmínticas com ivermectin e fenbendazole em equinos: Exames coprológicos e hematológicos. Rev. Bras. Parasitol. Vet., v.2, n.1, p.61-64, 1993.

UHLINGER, C.A. Use of fecal egg count data in equine practice. Comp. contin. Educ. Pract. Vet., v.15, n.5, p.742748, 1993. 
